\title{
Challenges of UAVs Adoption for Agricultural Pesticide Spraying: A Social Cognitive Perspective
}

\author{
Hsieh, Ting-Chu ${ }^{1}$, Hung, Ming-Chien ${ }^{2}$, Chiu, Mai-Lun ${ }^{3, *}$ and Wu, Pay-Jiing ${ }^{4}$ \\ 1 Nanhua University, Department of Information Management, No.55, Sec. 1, Nanhua Rd., Dalin \\ Township, Chiayi County 62249, Taiwan; tchsieh@mail.nhu.edu.tw \\ 2 Nanhua University, Department of Information Management, No.55, Sec. 1, Nanhua Rd., Dalin Township, \\ Chiayi County 62249, Taiwan; chemy@nhu.edu.tw \\ 3 National Penghu University of Science and Technology, Department of Information Management, \\ No.300, Liuhe Rd., Magong City, Penghu County 88046, Taiwan; mlchiu@gms.npu.edu.tw \\ 4 Nanhua University, Department of Information Management, No.55, Sec. 1, Nanhua Rd., Dalin Township, \\ Chiayi County 62249, Taiwan \\ * Correspondence: mlchiu@gms.npu.edu.tw
}

\begin{abstract}
Unmanned aerial vehicles (UAVs) are one of the most promising innovative technologies invented in recent years to promote precision agriculture and smart farming. UAVs can not only reduce labor requirements but also increase production output, reduce the use of pesticides, and protect the environment. However, previous studies on agricultural UAVs have mostly focused on technical problems such as software and hardware design. Few studies have examined users' behaviors in the implementation process. On the basis of Orlikowski and Gash's technological frames, this study explored the participants' cognition and expectation of famers, pesticide, sprayers, and agriculture officials, who are three key groups of stakeholders involved in the application of UAVs to pesticide spraying, regarding agricultural UAVs and examined how the conflicts between their cognition and expectation influenced the choice of using pesticide spraying UAVs. The conclusions of this study contributed to supplement the content and broaden the scope of application of technological frame theory and provided a crucial reference for the promotion of agricultural UAVs in practice.
\end{abstract}

Keywords: Unmanned aerial vehicles, Smart farming, Precision agriculture, Technological frames, Case study

\section{Introduction}

Unmanned aerial vehicles (UAVs), also known as drones or unmanned aircraft systems, are one of the most promising innovative technologies created in recent years [1]. UAVs are highly mobile and the images they produce are superior to satellite images and aerial photographs in ground resolution (or ground sampling distance) [2]. The recent development of relevant core technologies has led to safer, more secure, and more affordable UAVs, expanding the scope of application of civil UAVs to rescue operations, disaster management, forest monitoring, meteorological detection, green energy industry, and in particular, the agricultural industry [2-3]. [4] forecasted that the global UAV market will increase to US $\$ 52.3$ billion by 2025 from US\$20.71 billion in 2018 at a $14.15 \%$ compound annual growth rate (CAGR). In 
addition, the global market of UAVs for agricultural use was forecasted to grow from US $\$ 1.2$ billion in 2019 to US\$4.8 billion in 2024 at a 31.4\% CAGR [5]. This trend highlights the considerable demand for UAVs in the agricultural industry.

To increase the production volume of staple foods and mitigate farm labor shortage, countries worldwide have extensively promoted the concepts of precision agriculture and smart farming, emphasizing the use of emerging technologies such as robots, sensors, artificial intelligence (AI), big data analytics, and Internet of Things (IoT) to increase the production and sale of agricultural products. Among these innovative technologies, UAVs are considered a new-generation tool for promoting precision agriculture and smart farming [6-8]. UAVs combining various types of sensors and AI technologies have been widely applied in a broad range of agricultural activities (e.g., pesticide and fertilizer spraying, seed sowing, large-area crop loss assessment, production estimation, and pest detection) [9,7], thus simultaneously boosting the development of other new agricultural technologies.

Agricultural UAVs not only minimize labor requirements and cut down production costs [3], they also facilitate accurate pesticide spraying, which ensures the safety of agricultural products and the environment and at the same time reduces the risks associated with the use of chemical pesticides. Research related to the agricultural applications of UAVs has overly focused on software and hardware technologies rather than on users' behavior and psychology. To investigate the applications, challenges, and future research directions of civil UAVs, [3] mostly reviewed literature on the technical problems that could surface in the deployment of UAVs in precise agricultural applications, including soil texture mapping, plant disease detection, and crop maturity mapping. Shakhatreh et al. also indicated that machine learning and image preprocessing are the current topics of focus in research on the precise agricultural applications of UAVs. In addition, [7] summarized the precise agricultural applications of UAVs by reviewing technical studies that were conducted on UAV controllers, sprinkling systems, and geographic indicators such as the normalized difference vegetation index. [1] reviewed a broad range of studies investigating the agricultural and forestry applications of UAVs over the past 20 years. They reported that the topics of those agricultural studies were also centered on technologies related to precise agriculture and crop monitoring. Other studies such as [8-9] have also highlighted the extensive focus of agricultural UAV research on technical aspects. [11] was one of the few studies to examine the psychological behaviors of UAV users. However, Zheng et al. adopted statistical survey methods to identify the potential factors that influence the adoption of agricultural UAVs. These factors include gender, agricultural income ratio, understanding of new technologies, perceived ease-of-use, and perceived usefulness.

Social cognitive theory postulates that the adoption of information technology (IT) derives from social processes such as ongoing conflicts and mediation between participants [12-14] and is achieved collaboratively by the participants through the expression of different ways of thinking [15]. [16] also indicated that user expectation profoundly influences the adoption of IT and that this expectation is not only a factor influencing attitudes toward IT but also a key determinant of whether to adopt IT. On the basis of this concept, [17] proposed the technological frame (TF) theory, asserting that when organizations promote the adoption of IT, different stakeholders are actually involved in the process. Because of individual differences such as the nature of work, culture, and values, stakeholders tend to form specific cognitions and viewpoints regarding IT, which are referred to as TFs. Given this theoretical basis, Orlikowski and Gash studied a groupware consulting firm in greater depth and analyzed the TFs 
adopted in the process of IT adoption, subsequently revealing three domains of TFs - nature of technology, technology strategy, and technology-in-use. They further interpreted the resisting behaviors during the process of implementation on the basis of the incongruence between the three TFs among various groups. Since then, numerous studies have employed the TF theory to explain the adoption of different technologies and in turn revealed new TFs [18-23].

The technological functions of UAVs are becoming increasingly powerful, and the scope of UAV applications in the agricultural industry is expanding. In reality, farmers are not necessarily accepting of UAVs and are even constantly resistant to the adoption of this technology. The promotion of UAVs is often not as successful as expected [24]. Therefore, to facilitate the promotion of UAVs in the agricultural industry, the underlying reasons behind users' behavior should be explored from users' perspective rather than by focusing on technological aspects. According to social cognitive theory, the promotion of agricultural UAVs is analogous to the adoption of IT in that it is the outcome of ongoing conflicts and mediation among all participating groups expressing their viewpoints about agricultural UAVs. This process involves a complex and ever-changing phenomenon that is most effectively explored using qualitative research approaches. Therefore, this study was based on TF theory. The research participants included were farmers, pesticide sprayers, and agriculture officials in Yunlin, Taiwan, involved in the adoption of UAVs for pesticide spraying. The objective of this study was to determine the TFs of these three key stakeholders, that is, their cognition and expectation regarding agricultural UAVs, and how the conflicts between their cognition and expectation influenced the effective promotion of UAVs for pesticide spraying.

\section{Materials and methods}

\subsection{Research design}

This study adopted the longitudinal case study method. [25] stressed that the case study approach is particularly applicable to exploring contemporary phenomena within real-world contexts, specifically when the relationship between the research phenomenon and context is ambiguous, and when researchers hope to understand the "how" and "why" based on a diverse range of data. By analyzing TFs, the objective of this study was to understand the "why" - why was a powerful tool such as UAV not successfully implemented - and the "how"-how did the cognitive differences between the three key groups of stakeholders (farmers, pesticide sprayers, and government officials from the agriculture department) regarding UAVs lead to resistance to the adoption of UAVs, causing policy implementations to be not as effective as expected.

Onsite observations began in November 2017 and ended in May 2019, for a period of roughly 15 months. The subjects of observation were members of the three key groups of stakeholders involved in the promotion and adoption of UAVs for pesticide spraying in Yunlin, Taiwan. Data were collected by gathering historical data and making observations onsite. According to [26], people's ability to express themselves verbally in everyday life is derived from and grounded on their cognitive framework. Therefore, the simplest method to uncover people's default views about UAVs in their subconscious mind was to directly analyze the different media (e.g., verbal dialogue or written documents) used to communicate their thoughts. The corresponding data were collected using the following approaches:

(1) In-depth interviews: Unstructured interviews were conducted with two government officials from the agriculture department (manager of a farmers' association and clerk of a 
township hall), four pesticide sprayers, and four farmers, all of whom were members of the three key groups involved in the promotion and adoption of agricultural pesticide spraying UAVs in Yunlin. Each interview lasted 30-90 minutes, and interview data were either in the form of audio recordings or a written summary. During data analysis, OF1 and OF2 represented the two government officials from the agriculture department (hereafter referred to as "agriculture officials"); FR1-FR4 represented the four farmers; and SP1-SP4 represented the four pesticide sprayers.

(2) Observations: Observations were made and documented during coordination meetings, seminars, and demonstration activities related to the promotion and adoption of agricultural pesticide spraying UAVs in Yunlin. The objective was to observe different group members in a natural setting and determine the interpretations of each group about UAVs expressed through interactions, metaphors, and stories in the relevant situational context.

(3) Collection of data from documents: All types of data documenting the promotion of UAV pesticide spraying were collected and included meeting minutes, laws and regulations, reports, and other relevant media information. The cross-validation of data was achieved by collecting and analyzing a wide range of data.

\subsection{Data analysis}

In this study, the data were analyzed by following the TF analysis procedures proposed by [17], that is, separating data into groups, sorting group data into categories, identifying the common themes across groups, and identifying the TF domains. The four-step approach is described thereafter.

(1) Separating data into groups: The collected data are separated by key groups. That is, the data are grouped according to which key group's actions or statements they reflect.

(2) Sorting data into categories within the different groups: For each of these groups, interview transcripts and field notes are examined to identify the statements or actions that reflect the group's assumptions, knowledge, or expectations of a technology.

(3) Identifying the common themes across groups: The categories generated by each group's data are compared to determine the main categories or themes representing all members' assumptions, knowledge, or expectations of UAVs. Once the common themes are identified, the data of each group are re-examined using the proposed themes to identify the set of themes that covers most data.

(4) Identifying the TF domains: The themes are further classified, generating the domains of TFs. 


\subsection{Reliability and validity}

The quality, reliability, and validity of this research were improved through triangulation and examination of the interview process. Subsequently, member checks and peer examination were adopted to enhance the internal validity and reliability of this study.

Transcript reliability and validity. To improve the quality of the transcripts, the researcher transcribed the audio recorded data immediately after group discussions or interviews and incorporated the interviewees' facial expressions or actions from memory or according to notes (words and symbols) taken during the interview. Without changing the original meaning, the researchers deleted meaningless modal particles and pleonasms to improve readability.

Triangulation. This study adopted different data collection methods, including in-depth interviews, observations, field notes, and other secondary methods. In addition, a diverse range of data was collected. For example, the same theme was discussed with different interviewees. During data transcription and examination, other research personnel were invited to verify the data to further enhance data reliability.

Member checks. Once the audio recorded data were transcribed and observations documented, the relevant study participants were invited to check data correctness and completeness before subsequent data analysis was performed. Similarly, after the data were analyzed and reports compiled, the study participants were also invited to review the appropriateness and correctness of the study results.

\section{Case Background}

\subsection{Agricultural environment in Taiwan}

Taiwan is a densely populated island. Farmlands in Taiwan are sporadically scattered across the island, with each family farm having access to only roughly one hectare of cultivatable land. Because farm works cannot be performed on a large scale, the cultivation of crops often requires considerable labor input. In recent years, Taiwan has been plagued by serious population problems such as a low fertility rate, aging population, and workforce shortage. Therefore, the agricultural industry in Taiwan, which is mainly composed of small farm households, is still facing challenges in terms of sustainable development.

To address the challenges faced by the agricultural industry, Taiwan's agricultural sector has begun implementing the "Smart Farming" project since the end of 2016. The purpose of this project was to introduce advanced technologies (e.g., information communication technology, IoT, intelligent robots, big data analytics, and block chain) into the agricultural industry by combining governmental, academic, and industrial forces. The ultimate goal was to solve the problem of farm labor shortage and more importantly transform the production and sales model of Taiwan's agricultural industry through the integration of smart production and management. [27] described "smart farming" in Taiwan as a scene in which "a drone soars above the farmland to monitor crop growth and send data to cloud server, which then computes the amount of pesticide and chemical fertilizers that incurs minimal cost and environmental impact and determines the most effective practice for managing water resources; farmers can easily 
patrol their farm simply by connecting to the cloud server on their mobile phone or tablet computer (p.11)."

Currently, UAVs are one of the key innovative technologies promoted by the "smart farming" policies of Taiwan government's agriculture department. UAVs are slowly promoted and implemented through the development of relevant laws and regulations and research on the use of agricultural pesticides. On April 3, 2018, the legislative department of Taiwan's government approved amendments to the Civil Aviation Act, which added rules and regulations on the use of UAVs. Prior to the official implementation of those laws and regulations in 2020, the government's agricultural department is collecting the opinions of different parties, with the hope of introducing regulations exclusive to the adoption of agricultural UAVs and closing the gap between theory (the Civil Aviation Act) and practice (existing farmers' adoption of agricultural drones) to facilitate the promotion of UAVs in the agricultural industry. To ensure the safe use of agricultural pesticide spraying UAVs, the Council of Agriculture established the agricultural drone project in 2018. Members from the Agricultural Chemicals and Toxic Substances Research Institute, Taiwan Agricultural Research Institute, and Bureau of Animal and Plant Health Inspection and Quarantine were gathered to form an agricultural drone research team. The team conducted large-scale studies on the application of drones in pesticide spraying, evaluated the safety of agricultural drones for pesticide spraying, and finally established a complete system for managing agricultural pesticide spraying drones and pesticide use. The findings should improve the operating safety of pesticide sprayers and ensure farmers' safe use of pesticides (Chiang et al.,2018).

\subsection{Agricultural status of Yunlin and measures for promoting smart farming}

Yunlin County is the largest agricultural zone in Taiwan located in the central-south region of Taiwan and at the northern tip of the Chianan Plain. The county is populated by approximately 680,000 people ( $2.9 \%$ of Taiwan's total population), $40 \%$ of whom are employed in the agricultural industry. The area of Yunlin County is roughly 129,000 ha (3.6\% of Taiwan's land), and 81,000 ha of the area is cultivatable land (62\% of Yunlin's total area). The county has a subtropical climate, which is suitable for agriculture, forestry, fishing, and animal husbandry industries. Specifically, Yunlin County is the largest producer of rice, vegetables, garlic, potatoes, and peanuts in Taiwan. According to the 2018 statistical yearbook of the Council of Agriculture, rice accounts for the largest part of harvested agricultural products in Yunlin County. Yunlin produces approximately 46,000 ha of rice, the largest area in Taiwan, accounting for $19.1 \%$ of the total area of harvested rice across Taiwan, followed by 36,944 ha of vegetables, which accounts for $27.46 \%$ of the total harvested area across Taiwan.

The county is facing serious problems in terms of agricultural population aging and farm labor shortage. In response, the local government has decided to introduce and implement advanced, automated, and smart farming machineries and technologies. Since 2006, Yunlin County has hosted the Agricultural Machinery and Materials Exhibition every year, which is the largest agriculture-related exhibition in Taiwan. The objective of this exhibition is to keep farmers abreast of new agricultural technologies. Over the past few years, the local government has also cooperated with the agricultural sector to promote the "Smart Farming" policy and actively transform Yunlin into an "agricultural capital." By introducing UAVs and other innovative technologies, the government intends to strengthen farmers' competitiveness and attract young adults to engage in agricultural production, thereby driving the transformation of 
agricultural production to facilitate the sustainable development of the agricultural industry in Yunlin.

\section{Analysis and Findings}

\subsection{TFs of the key groups}

The different data collected in this study were examined, classified, and summarized. Subsequently, three TF domains were established, and they are described in detail as follows.

Technological capabilities: This domain refers to each key group's cognition and understanding of the functionality and capabilities of UAVs in pesticide spraying. For example, "using a drone is an indirect way of spraying pesticides (remote-controlled), which benefits both us and farmers... [because the drone] sprays concentrated aerosol, it is definitely more efficient [than the conventional pulley-type sprayers...]" (SP1).

Technological strategies: This domain refers to each key group's understanding of the underlying motivations, visions, and potential outcomes of a governmental policy that promotes the adoption of UAVs for pesticide spraying. For example, "promoting it [pesticide spraying UAVs] is aimed at changing the traditional mode of farming operations of the entire agricultural industry. For a long time, our farmers have made no changes [to farming methods] and are prone to become outdated, given the rapid development [of foreign technologies]..." (OF1).

\subsection{Regulations of related technology}

This domain refers to each key group's understanding of the laws and regulations and problems associated with the adoption of UAVs for pesticide spraying. For example, "... requiring certification to use UAVs is a good thing for business; [it also means that] not anyone can spray pesticides, which is a form of recognition for us and a type of protection for them [farmers]..." (SP4).

Each domain represents a group of highly associated assumptions, expectations, and knowledge, serving as a basis for all users of pesticide spraying UAVs to understand the adoption of UAVs. During the analysis, although the TFs were classified into different domains, these domains remained associated with each other and influenced each other [19]. For example, when farmers demonstrated skepticism regarding the functionality and capabilities of UAVs, they not only communicated their views about "technological capabilities" but also complained about the use and legal aspects of UAVs, thereby leading to expectations regarding the "regulations of relevant technology." In the next section, the three TF domains obtained in this study were used to further analyze the incongruence between the three key groups' TFs in the adoption of UAVs for pesticide spraying in Yunlin and to examine how such cognitive incongruence influences the promotion of pesticide spraying UAVs.

\subsection{Incongruence between TFs and its effect on the application of UAVs in pesticide spraying}

In three episodes, this study described how the incongruence between the TFs of the three key groups (farmers, pesticide sprayers, and agriculture officials) influenced their adoption of UAVs when the use of UAVs in pesticide spraying was promoted in Yunlin under Taiwan's 
smart farming policy. In the descriptions, words in italics are used to indicate the TF domains in each plot.

\section{Episode 1: Promotion of the policy to cut the use of chemical pesticides by half within a decade.}

To ensure the safety of staple foods and agricultural products and comply with the international standards for pesticide risk mitigation, in September 2017, the Council of Agriculture in Taiwan implemented a policy aimed at reducing the use of chemical pesticides by half within a decade through 12 measures, including building a pesticide safety classification management mechanism, improving the quality of pesticide products, and eliminating hazardous chemical pesticides. One of the 12 measures also involved actively promoting the original pesticide spraying system and amending the "Regulations for Training Contracted Pesticide Applicators". In 2019, UAV pesticide spraying training was introduced in training programs for the first time to address the farm labor shortage problem, strengthen the safety of pesticide use, and achieve the pesticide reduction goal.

In general, agriculture officials were optimistic about the policy on the use of UAVs to aid pesticide spraying activities and reduce the use of chemical pesticides. The officials indicated that using UAVs integrated with various types of sensors not only facilitates the accurate spraying of pesticides but also greatly reduces the use of pesticides, both of which are conducive to the implementation of this policy (technological capabilities and technological strategies). However, from farmers' perspective, the first thing they considered was not whether the use of pesticide could be reduced but whether using pesticide spraying UAVs could achieve the desired outcome (technological strategies). The officials, pesticide sprayers, and farmers expressed their opinions regarding the policy as follows:

“...using drones to spray pesticides would definitely benefit [the implementation of] this policy!...to effectively implement this policy, existing practices [pesticide spraying] must be thoroughly reviewed. When the conventional method is applied under the same conditions, the types and dose of pesticide only increase, not decrease. The resulting reduction is most significant when UAVs are used..." (OF1)

"To be honest, they [farmers] are impassive regarding this pesticide reduction policy... with the number of pests, they want to spray as much pesticides as possible! I know that with UAVs, [the use of pesticide] can be reduced...because a flying UAV generates airflow that exposes the lower side of the leaf to the pesticide, yielding better outcome. You have to show them that it [UAVs] is effective, which is the most important, otherwise they will not use it!" (SP1)

“...Using less pesticides is a good thing, we know that. But are UAVs really that effective? They said it [UAV] can cut the use [of pesticide] by half, that is quite unlikely!..." (FR4)

“...now they are using UAVs for spraying pesticides as well. They simply dilute it [pesticide] with water. The amount of money spent on pesticides is roughly the same. So I think reducing the use will not be that easy. It [use of UAVs] probably saves labor costs..." (FR1)

Evidently, the farmers were more concerned about whether using UAVs to spray pesticides can truly achieve the desired effect. They were indifferent toward or not confident regarding the reduction by half of pesticide use. By contrast, the agriculture officials believed that using UAVs for pesticide spraying can not only reduce the use of pesticides but also change the conventional method of pesticide spraying (technological strategies), increase the safety of 
pesticide use (regulations of relevant technology), and minimize the environmental impact of pesticides (technological strategies).

“...when using drones to spray pesticides, the applicator or farmers themselves will not come into direct contact with the pesticide. [This method] is safe for people; ...lower doses are used, and pesticides are not sprayed directly onto the soil. [This method] causes minimal damage to the soil! (OF1)

"....in future, pesticides exclusively for UAVs [will be developed]; these pesticides will be less toxic and more effective. By then, the use of pesticides will be reduced considerably, and UAVs will be more widely implemented." (OF2)

Although the government's agriculture department intends to use safety and health reasons to persuade farmers to adopt pesticide spraying UAVs, and pesticide sprayers can guarantee the effectiveness of pesticide spraying UAVs, the farmers were skeptical about the functions and benefits of UAVs (technological capabilities) and concerned about subsidy problems (regulations of relevant technology), which affected their intention to use UAVs. Farmer A explained why they were concerned and resistant toward using UAVs:

“...I'm not quite sure about this spraying technique. I think our pulley-type sprayer is fine. I suppose I could try out the new method..." (FR2)

"Subsidies...subsidies are very important. Seems like [we] do not see much of it. Yes using UAVs will protect users from skin contact with pesticides. However, most [farmers] are hiring companies to do it [spray pesticide]. These companies are quite well equipped. Without subsidies, everyone might be less willing to use UAVs..." (FR4)

Concerning the promotion of the pesticide reduction policy, the agriculture officials hoped to tap into the power of innovative technologies such as UAVs to cut the use of pesticides by half and change the farmers' habits of spraying pesticides. However, farmers appear to be more concerned about whether the efficiency of UAVs is exactly as advertised and whether UAVs can effectively spray pesticides at a lower cost. This cognitive difference regarding the technical capabilities of UAVs and the value of such policy implementation affected the adoption of UAVs for pesticide spraying.

\section{Episode 2: Spreading of periodical rice blast disease.}

Rice in Taiwan is harvested twice a year, from February to July (first crop season) and from August to January (second crop season). The first crop season begins when spring transitions to winter. During this period, the climate is humid and varies considerably, which easily leads to rice blast infection. Every year during this period, rice blast affects more than $10 \%$ of the total planting area. In March 2018, the government's agriculture department in southern Taiwan announced the spreading of rice blast disease in rice paddy fields and advised farmers to strengthen their field management and take precautionary measures as soon as possible (e.g., pesticide spraying for preventive purposes or intensive spraying of pesticides on infected fields). Under this urgent context, gathering sufficient labor force in a short time to assist with management and mass spraying of pesticides became a major challenge in rice blast prevention. Given the efficiency features of UAVs (fast and saves time and labor; technological capabilities), they became an extremely useful tool for aiding in the prevention of rice blast disease (technological strategies) and are accepted as such by agriculture officials, pesticide sprayers, and farmers: 
“...Spraying pesticides with drones is most adequate because immediately after a disease outbreak, the most pressing matter is spraying pesticides intensively, systematically, and comprehensively...hiring someone to do it is not easy. Using drones can indeed solve this problem." (OF2)

"... One advantage of using drones is cutting down cultivation costs, which in turn generates more profit for them [farmers]. Another advantage is controlling the use of pesticides, including the type of pesticide used, formula, and even the toxicity of pesticide residues, which provides an additional layer of protection for agricultural crops..." (OF1)

"We can spray pesticides very intensively (disease is effectively controlled only after spraying pesticides two to three times) and also properly plan our schedules...it [drone] works fast...it [drone] is an excellent solution to labor shortage problems..." (SP2)

"Using drones to spray pesticides sounds like a good idea. They made it sound so powerful when they introduced it...it is now the first crop season when rice blast easily develops. Seeking help at the last minute is not easy. If only information [about rice blast] could be released earlier. Now we do not have much choice!" (SP4)

When rice blast occurs, UAVs provide pesticide sprayers with a new option for spraying pesticides. In the case when farmers are unable to find sufficient help but have to spray crops intensively with pesticides in a short timeframe, they took the advice of pesticide sprayers and government officials and adopted UAVs. However, they actually had reservations about the function and potential benefits of this new technology (technological capabilities), and they were also skeptical about the policy implementation (technological strategies). According to the farmers and pesticide sprayers:

"Drones carry so little water. We can spray a lot more pesticide with our pulley pipes. Do drones actually work?...If not, we might as well do it [spray] ourselves..." (FR3)

"[UAVs] may be as powerful as they say, but they are similar to standard farm machines. Our pulley pipes are not that bad, they can spray more [pesticides] and are easier to control..." (FR3)

"...I know they [farmers] think that spraying too little [pesticide] will be ineffective. Actually, the pesticide used in this machine [UAVs] is different...maybe if the price was lower, [UAVs] it will be more attractive to them. But we need quantity in order to reduce the price." (SP2)

Based on the aforementioned interviews, pesticide sprayers can provide sufficient technical support. However, cost is still their main factor of consideration. Farmers generally felt safer using traditional pesticides and conventional spraying techniques and were not confident with using UAVs. They even perceived UAVs as an ordinary farming machinery, which in turn affected their intention to use UAVs.

\section{Episode 3: Implementation of UAV management regulations.}

In response to the vigorous development of UAV industries and applications, the Taiwanese government has actively legislated regulations for UAVs. On April 3, 2018, Taiwan government's legislative department officially approved amendments to the Civil Aviation Act, which added a set of regulations designed specifically for UAVs. The amendments marked the first time that UAVs are included in aviation management and are expected to be promulgated at the beginning of 2020. Before that, the government will try to facilitate policy implementation. To that end, they plan on organizing educational propaganda, establishing 
mechanisms for coordinating administration works between the central and local governments, and building UAV management information systems as well as an app that provides maps and information on activity areas in Taiwan.

The amended Civil Aviation Act stipulates clear rules on the management of UAVs, including UAV registration, inspection, operator certification, activity areas, restrictions of operation, flight activity application, and insurance. The amended regulations also directly influence the use of UAVs in the agricultural industry. For example, the amendments mandate that drones with a maximum takeoff weight exceeding $250 \mathrm{~g}$ must be registered, that operators of drones with a takeoff weight exceeding $250 \mathrm{~g}$ must hold a license to operate UAVs, and that operators of UAVs must be government agencies (institutions), schools, or legal persons. In other words, farmers cannot operate UAVs arbitrarily. Specifically, when using UAVs to spray pesticides or fertilizers, farmers must file applications with the government, pursuant to regulations. The agriculture officials exhibited a positive attitude toward the amended UAV management regulations. They believe that these amendments are beneficial to the future development of the agricultural industry (regulations of relevant technology and IT policy):

"Having a clear set of regulations is good. Everyone will know what to do. [I] often see news about drone accidents. See that huge machine spraying pesticides? Is it not dangerous? It's no joke if they fall. Laws must be in place so that civil servants know what to do. This is good for everyone..." (OF2)

"I know there are still some flaws in it [laws]. Some time is necessary to adapt...look at the United States, Japan, and even China, they all have it [laws], it would be unreasonable if we do not have it...I think the agricultural industry needs a reform. This is inevitable." (OF1)

Although the amended regulations ensure the safe use of UAVs and provide the government with a legal basis for managing UAVs, the amendments are not in line with the actual practices of farmers and pesticide sprayers. Farmer A and Pesticide Sprayer A both expressed their apprehension about the implications of the amended law (regulations of relevant technology):

"Requiring operators to hold a license is a good thing [because] it proves our expertise, but asking us to register etc. ...UAVs have become extremely popular over the past few years, but honestly speaking they are still in the growth phase. Most of them [farmers] are still not big believers [of UAVs], which makes me wonder whether our whole business will fail." (SP3)

"In my opinion, the government does not have a complete set of policies and regulations for the management and implementation of pesticide spraying UAVs. To be honest, this [problem] is giving us business owners a headache...we are still unclear as to whether UAVs will be listed as an agricultural machinery and whether subsidies will be provided..." (SP1)

“...it is fine for now, but in the future, if [the government] imposes stricter control over pesticide spraying UAVs to a point that inconveniences us, we might consider not using it [UAVs]...take this as an example, my farmland is this big, if something unexpected happens and I need to spray pesticides urgently, an application takes 15 days to process, by then my crops will most likely be dead..." (FR4)

"In fact, some of us are also thinking about whether to get one [UAVs] ourselves. UAVs are not subsidized because they are not an agricultural machinery, which discourages everyone to get them... Now you either need a license or you must be a legal person etc. in order to operate UAVs. In that case, it [obtaining UAVs] is definitely unlikely to happen." (FR1) 
There is still some time before the amended Civil Aviation Act comes into effect, and the agriculture officials indicated that they will collect opinions from all parties and amend the Civil Aviation Act or establish implementation rules for agricultural UAVs accordingly. However, the pesticide sprayers and farmers remain skeptical regarding the amendments and regard the amendments as an obstacle to promoting pesticide spraying UAVs that occasionally affects their intention to use UAVs.

In these three episodes, this study identified incongruence between the TFs of different key groups. In other words, the key groups exhibited considerable cognitive difference and prejudice against the adoption of UAVs, which in turn affected their use of UAVs in pesticide spraying.

First, regarding the cognition of "technological capabilities," the pesticide sprayers believed that UAVs are associated with numerous novel techniques that can be introduced and applied to the agricultural industry to facilitate farming activities. By contrast, the agriculture officials regarded UAVs as an innovative technology that brings about change in the agricultural industry, similar to Markus and Benjamin's (1997) “magic bullet." Conversely, the farmers perceived UAVs as another type of agricultural machinery and a "tool" that can improve their work efficiency.

Second, regarding the cognition of "technological strategies," the agriculture officials hoped that through promoting the adoption of UAVs in pesticide spraying, the use of pesticides can be reduced or labor shortage problems and plant disease prevention can be addressed. Moreover, they hoped that technology can act as a promoter that brings about change in the agricultural industry to transform Taiwan's agriculture into smart agriculture. However, the farmers appeared to be unaware of the implications of this policy. Instead, they were more concerned about whether the functionality of UAVs was effective or whether UAVs were a cost-saving solution. Similarly, pesticide sprayers were also concerned about the functional and technical aspects of the technology to satisfy the demands of their consumers.

Finally, regarding the cognition of "regulations of relevant technology," farmers and pesticide sprayers all believed that introducing laws for UAVs imposes a form of restriction on the use of UAVs in the agricultural industry. Particularly, a wide gap exists between theory (law) and practice (farming practices), and this gap influences user intention. However, the agriculture officials believed that legal regulations are necessary because in the long run, laws can facilitate the promotion of UAVs in the agricultural industry. Nevertheless, before the official implementation of the amended Civil Aviation Act, the government will slowly introduce regulations for the adoption of UAVs in pesticide spraying and other agricultural applications. Table 1 summaries the TFs of each key group. 
Table 1. TFs of key groups.

\begin{tabular}{|c|l|l|l|}
\hline stakeholder & \multicolumn{1}{|c|}{ Farmer (FR) } & Pesticide Sprayer (SP) & $\begin{array}{l}\text { Government Official } \\
\text { (OF) }\end{array}$ \\
\hline $\begin{array}{c}\text { Capabilities of } \\
\text { Technology }\end{array}$ & $\begin{array}{l}\text { Regards UAVs as a } \\
\text { "tool" for improving } \\
\text { farm work efficiency } \\
\text { and as an ordinary } \\
\text { agricultural } \\
\text { machinery. }\end{array}$ & $\begin{array}{l}\text { Regards UAVs as a } \\
\text { new technology that } \\
\text { can be introduced to } \\
\text { help farmers improve } \\
\text { their farming practices } \\
\text { and work efficiency. }\end{array}$ & $\begin{array}{l}\text { Regards UAVs as an } \\
\text { "innovative } \\
\text { technology" that } \\
\text { brings about change in } \\
\text { the agricultural } \\
\text { industry, such as a } \\
\text { "magic bullet." }\end{array}$ \\
\hline $\begin{array}{c}\text { Technology } \\
\text { Strategies }\end{array}$ & $\begin{array}{l}\text { To solve labor } \\
\text { shortage problem and } \\
\text { reduce pesticide use. }\end{array}$ & $\begin{array}{l}\text { To solve labor } \\
\text { shortage problem and } \\
\text { reduce pesticide use. }\end{array}$ & $\begin{array}{l}\text { To implement the } \\
\text { transformation of the } \\
\text { agricultural industry } \\
\text { and promote "smart } \\
\text { farming." }\end{array}$ \\
\hline $\begin{array}{c}\text { Regulations of } \\
\text { Technology }\end{array}$ & $\begin{array}{l}\text { Laws impose a form } \\
\text { of restriction on the } \\
\text { agricultural } \\
\text { applications of UAVs. }\end{array}$ & $\begin{array}{l}\text { Laws impose a form } \\
\text { of restriction on the } \\
\text { agricultural } \\
\text { applications of UAVs. }\end{array}$ & $\begin{array}{l}\text { Laws are necessary } \\
\text { and facilitate the } \\
\text { long-term } \\
\text { development of UAVs } \\
\text { in agricultural } \\
\text { application. }\end{array}$ \\
\hline
\end{tabular}

\section{Conclusions}

Through the collection and analysis of qualitative data, this study first developed three TF domains, namely, technological capabilities, technological strategies, and regulations of relevant technology. Subsequently, the three domains were employed to further analyze the cognition of the three key groups (farmers, pesticide sprayers, and agriculture officials) and present their fundamental cognitive differences regarding the promotion of UAVs in pesticide spraying (TF domains), and how this cognitive difference resulted in resistance toward the adoption of UAVs, subsequently causing policy implementation to be not as effective as expected. The theoretical implications and contributions of the results of this study are described thereafter.

\subsection{Contributing to TF theory.}

The developed TFs were nested in a situational context. For the different case scenarios studied, the technology analyzed was different, which may have led to different TF domains and contents. The scenarios examined in this study have not been discussed in previous related studies. Therefore, even if the TF domains and contents in this study have been presented in previous research findings, this study still contributed to the theoretical implication of TF domains, particularly in the "regulations of relevant technology." Although the term "regulations of relevant technology" is similar to the "IT-in-use" domain proposed by [17], it emphasizes the legal aspect of a technology and also reflects that the use and promotion of a new technology such as UAVs must be regulated by law, in contrast to business information 
systems or IT discussed in previous studies. Therefore, the TF domains developed in this study contributed to TF theory.

\subsection{Increased the explanatory power of TF theory.}

General information systems were typically the main focus of previous TF studies, such as $[28,19]$, or [18], which all adopted general information technologies. This study was the first to apply TF analysis to an agricultural setting and adopt a new innovative technology, UAVs, as the research subject. This study provided a full explanation of the causes of problems and of the promotion process of the use of UAVs in pesticide spraying. In addition, the explanatory scope of TF theory was expanded to include explanations on the resistance to the adoption of agricultural technologies. In future, TF theory can be used to explain problems in the adoption of other new agricultural technologies.

Finally, the results of this study also provided key practical implications that can serve as a reference for promoting the adoption of new technologies similar to UAVs. The promotion of "precision agriculture" and "smart farming" heavily relies on the use of innovative technologies. The research and development of techniques and functions of a technology are crucial. However, how to motivate farmers to accept and apply technology in farming activities is the key to successfully implementing "precision agriculture" and "smart farming." This study adopted social cognitive perspective to identify the reasons and process of resistance to the adoption of UAVs. When promoting the use of new agricultural technologies in future, the government or tech companies can focus on farmers' fundamental differences in their cognition regarding the three TF domains (technological capabilities, technological strategies, and regulations of relevant technology). They can then reconstruct a technology based on different frames to reduce prejudice and change the farmers' opinions about a technology, in turn positively influencing the farmers' behavior and greatly facilitating the promotion of "precision agriculture" and "smart farming."

Author Contributions: Formal analysis, Ting-Chu Hsieh; Investigation, Pay-Jiing Wu; Methodology, Ming-Chien Hung; Writing - review \& editing, Mai-Lun Chiu.

Funding: This study was received funded by Ministry of Science and Technology, Taiwan.

Acknowledgments: We thank the anonymous reviewers for their insightful comments to improve the clarity of the theoretical arguments in the manuscript.

Conflict of Interest: All of the authors declare that they have no conflict of interest.

\section{References}

1. Raparelli, E.; Bajocco, S. A bibliometric analysis on the use of unmanned aerial vehicles in agricultural and forestry studies. International Journal of Remote Sensing. 2019, 40, 9070-9083.

2. Singh, V.; Bagavathiannan, M.; Bhagirath. S.C. Evaluation of current policies on the use of unmanned aerial vehicles in Indian agriculture. Current Science. 2019, 117, 25-29.

3. Shakhatreh, H.; Sawalmeh, A., Al-Fuqaha, A. Unmanned aerial vehicles (UAVs): A survey on civil applications and key research challenges. IEEE Access. 2018, 7, 48572-48634. 
4. Markets and Markets. Unmanned Aerial Vehicle (UAV) Market by Vertical, Class, System, Industry, Type, Mode of Operation, Range, Point of Sale, MTOW and Region :Global Forecast to 2025. Available at:https://www.marketsandmarkets.com/Market-Reports/unmanned-aerial-vehicles-uav-m arket662.html?gclid=Cj0KCQjw0IDtBRC6ARIsAIA5gWvrXud5G91nAZksR1Wjqu2TnbfzW S3ihpcPAXXkOTu1ypmketMque4aAhYyEALw_wcB. (accessed October 2019).

5. Markets and Markets. Agriculture Drones Market by Offering, Application, Component, and Geography: Global Forecast to 2024. Available at:

https://www.marketsandmarkets.com/Market-Reports/agriculture-drones-market-2370976 4.html?gclid=Cj0KCQjw0IDtBRC6ARIsAIA5gWuH7Mvs13w9Mwt_GiDdU-pKQ9R0B SL7kF-cUIUeSIt6H0OBeamXgVYaAqimEALw_wcB. (accessed October 2019).

6. Hunt, R.; Daughtry, C. What good are unmanned aircraft systems for agricultural remote sensing and precision agriculture? International Journal of Remote Sensing. 2018, 39, 5345-5376.

7. Mogili, U.; Deepak, B. Review on application of drone systems in precision agriculture. Procedia Computer Science. 2018, 133, 502-509.

8. Pasquale, D.; Luca, D.; Luigi, G. A review on the use of drones for precision agriculture. IOP Conference Series: Earth and Environmental Science. 2019, 275, 012022.

9. Kim, J.; Kim, S.; Ju, C. Unmanned aerial vehicles in agriculture: A review of perspective of platform, control, and applications. IEEE Access. 2019, 7, 105100-105115.

10. Dragamestianos, C.; Messini, C.I.; Antonakis, P.T.; Zacharouli, K.; Kostopoulou, E.; Makrigiannakis, A., ... \& Daponte, A. The effect of metformin on the endometrium of women with polycystic ovary syndrome. Gynecologic and obstetric investigation. 2019, 84(1), 35-44.

11. Zheng, S.; Wang, Z.; Wachenheim, C. Technology adoption among farmers in Jilin Province, China. China Agricultural Economic Review. 2019, 11, 206-216.

12. Newman, M.; Noble, F. User involvement as an interaction process : A case study. Information Systems Research. 1990, 1(1), 89-113.

13. Hirschheim, R.; Newman, M. Symbolism and information systems development: Myth, metaphor and magic. Information Systems Research. 1991, 2(1), 26-62.

14. Bergman, M.; King, J.; Lyytinen, K. Large scale requirements analysis revisited: The need for understanding the political ecology of requirements engineering. Requirements Engineering. 2002, 7(3), 152-171.

15. Dougherty, D. Interpretative barriers to successful product innovation in large firms. Organization Science. 1992, 3(2), 179-202.

16. Ginzberg, M. Early diagnosis of MIS implementation failure: Promising results and unanswered questions. Management Science. 1981, 27(4), 459-478.

17. Orlikowski, W.; Gash, D. Technological frames: Making sense of information technology in organizations. ACM Transaction on Information Systems. 1994, 12(2), 174-207.

18. McLoughlin, I.; Badham, R.; Couchman, P. Rethinking a political process in technological change: Socio-Technical configurations and frames. Technology Analysis \& Strategic Management. 2000, 12, 17-37.

19. Davidson, E. Technology frames and framing: A Socio-Cognitive investigation of requirements determination. MIS Quarterly. 2002, 26(4), 329-358. 
20. Lin, A.; Silva, L. The social and political construction of technological frames. European Journal of Information Systems. 2005, 14, 49-59.

21. Puri, S. Technological frames of stakeholders shaping the SDI implementation: A case study from India. Information Technology for Development. 2006, 12(4), 311-331.

22. Davis, C.; Hufnagel, E. Through the eyes of experts: A Socio-Cognitive perspective on the automation of fingerprint work. MIS Quarterly. 2007, 31(4), 681-703.

23. Hsu, C. Frame misalignment: interpreting the implementation of information systems security certification in an organization. European Journal of Information Systems. 2009, 18(2), 140-150.

24. Freeman, P.; Freeland, R. Agricultural UAVs in the U.S.: Potential, policy and hype. Remote Sensing Applications: Society and Environment. 2015, 2, 35-43.

25. Yin, R. Case Study Research: Design and Methods, Los Angeles, CA: Sage. 2009.

26. Moch, M.; Fields, W. Developing a content analysis for interpreting language use in organizations. Research in the Sociology of Organizations. 1985, 4, 81-126.

27. Chen, C.; Yang, C. Promotion of smart farming: Flipping Taiwan agriculture. Public Governance Quarterly. 2017, 5, 104-111.

28. Lin, A.; Cornford, T. Framing Implementation Management. In: Proceedings of the 21st International Conference on Information Systems, Atlanta, USA. 2000, 197-205. 